\title{
Aberrant transcriptional regulation could explain phenotypic variability in autosomal recessive polycystic kidney disease
}

\author{
Friedrich C. Luft
}

Published online: 7 August 2014

(C) Springer-Verlag Berlin Heidelberg 2014

Autosomal recessive polycystic kidney disease (ARPKD) is an inherited ciliopathy of childhood caused by mutations in the PKHDI and is less frequent than the better-known ciliopathy, autosomal dominant polycystic kidney disease. Characteristic dilatation of the renal collecting ducts is prominent in ARPKD. The disease can start already in utero and presents at any stage from infancy to adulthood. Early renal insufficiency may develop in utero and can lead to early abortion or oligohydramnios (Potter's sequence). Half of these children die of pulmonary hypoplasia after delivery. However, children may also be born who have no evidence of renal dysfunction in utero and who have normal renal function at birth. A third of ARPKD patients die in the perinatal period, and those surviving the neonatal period commonly develop end-stage renal disease (ESRD) in infancy, early childhood, or adolescence. Some patients are first diagnosed as adults. While bilateral renal enlargement with microcystic dilatation is the predominant clinical feature (Fig. 1), arterial hypertension and intrahepatic biliary dysgenesis are also important manifestations. All patients with ARPKD develop congenital hepatic fibrosis. However, the biliary dysgenesis may result in nonobstructive dilation of intrahepatic bile ducts (Caroli's disease). Cholangitis and variceal bleeding develop in some patients necessitating a two-organ transplantation. Thus, the clinical spectrum of ARPKD is broad and heterogeneous. Büscher et al. [1] recently provided an excellent clinical review. The profound clinical variability can occur within families with the same genotype. Variable phenotypes could also result from differences in genetic background, combinations of mutations, modifying genes, epigenetic factors,

F. C. Luft $(\bowtie)$

Experimental and Clinical Research Center, Charité Medical Faculty, Berlin, Germany

e-mail: luft@charite.de hormonal effects, or environmental influences [2-4]. Chainterminating, truncating PKHD1 mutations result in more severe disease than amino acid substitutions [5, 6].

Alternative splicing is a regulated process during gene expression that results in a single gene coding for multiple proteins [7]. In this process, particular exons of a gene may be included within, or excluded from, the final processed messenger RNA (mRNA) produced from that gene. As a result, the proteins translated from alternatively spliced mRNAs will contain differences in their amino acid sequence and often in their biological functions. More than $90 \%$ of all human genes are alternatively spliced [8]. Alternative splicing allows the human genome to direct the synthesis of many more proteins than would be expected from its 20,000 protein-coding genes. However, alternative splicing within a given gene can also result in disease. For instance, the Parkinson protein 2, E3 ubiquitin protein ligase gene $P A R K 2$, is one of the largest genes in our genome [9]. The gene is implicated in the pathogenesis of autosomal recessive juvenile Parkinsonism, and it has been recently linked to cancer, leprosy, autism, type2 diabetes mellitus, and Alzheimer's disease. The PARK2 primary transcript undergoes extensive alternative splicing, which enhances transcriptomic diversification and protein diversity in tissues and cells. The PARK2 alternative splice transcripts and isoforms have been correlated with those in rat and mouse, two common animal models for studying human disease genes. Alternative splicing relies on complex processes that are easily altered by both cis- and trans-acting mutations. Although the contribution of PARK2 splicing in human disease remains to be fully explored, some evidences show disruption of this versatile form of genetic regulation may have pathological consequences.

In this month's issue of J Mol Med, Boddu and colleagues [10] studied alternative splicing of PKHD1. The human gene spans $469 \mathrm{~kb}$, has 86 exons, 15 of which exhibit alternative splicing boundaries. The mouse homolog, $P k d h 1$, is equally 


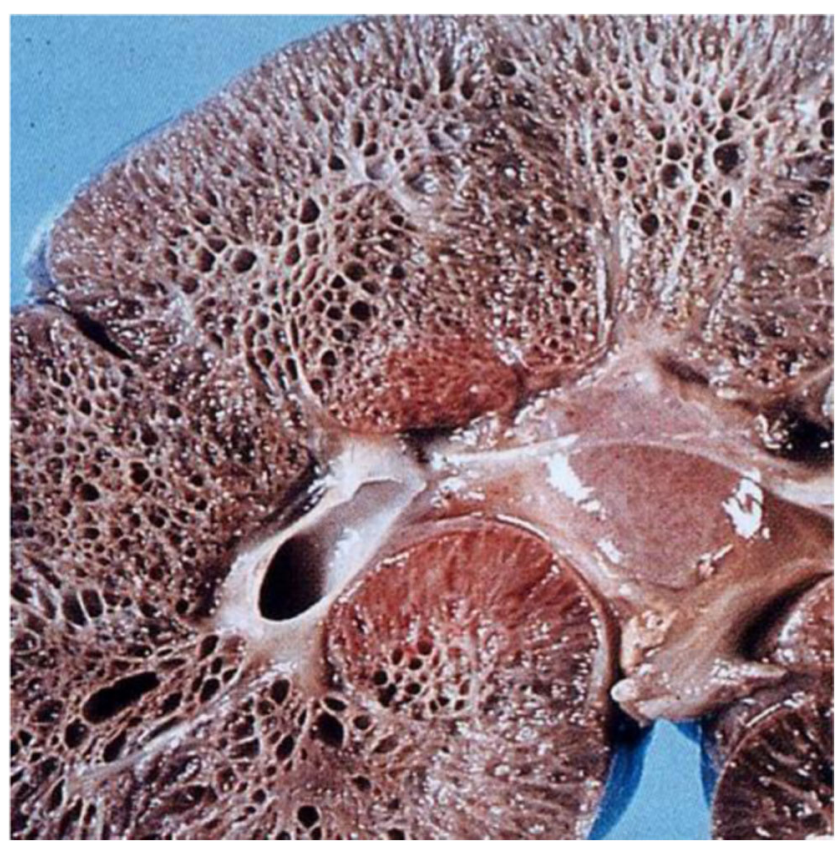

Fig. 1 ARPKD is a ciliopathy but afflicts primarily the collecting ducts. ARPKD kidneys have a sponge appearance with elongated cysts perpendicular to the cortical surface. The kidneys are large but remain smooth, different from autosomal-dominant polycystic kidney disease, another cilipathy. Both conditions feature involvement of other organs, including the liver, pancreas, and cerebral vessels. Both conditions cause hypertension and renal failure. ARPKD more commonly causes hepatic fibrosis, cirrhosis, and liver failure

complex and similar in structure. The authors characterized the kidney-specific transcriptional profile of $P k d h 1$ and found that $P k h d 1 / P K H D 1$ transcriptional processing is modulated by serine/arginine-rich splicing factor family intragenic motifs. Their analysis of PKHD1 missense variants suggests that dysregulated $P K H D 1$ splicing represents an unappreciated pathogenic mechanism in ARPKD. Boddu et al. [10] observed multiple alternatively spliced $P k h d 1$ transcripts that varied in size and exon composition in embryonic mouse kidney, liver, and placenta samples, as well as among adult mouse pancreas, brain, heart, lung, testes, liver, and kidney. They then used real-time PCR and RNA sequencing to identify 22 novel $P k h d l$ kidney transcripts with unique exon junctions. They observed various mechanisms of alternative splicing, including exon skipping, use of alternate acceptor/ donor splice sites, and inclusion of novel exons. They extended their findings with bioinformatics analyses and exontrapping experiments to validate consensus-binding sites for serine/arginine-rich proteins that modulate alternative splicing. Boddu et al. [10] then used site-directed mutagenesis to study the functional importance of selective splice-site enhancers. They then demonstrated that many of the novel transcripts were polysome-bound. Finally, they examined the human PKHD1 R760H missense variant. They found that this variant alters a splice enhancer motif that disrupts exon splicing and thereby truncates the protein. Aberrant $P K H D 1$ splicing evidently represents an unappreciated pathogenic mechanism in $A R P K D$, perhaps relevant to the heterogeneous phenotypes and their severity.

Pre-mRNA processing occurs with high fidelity and accuracy. Models have been proposed that define processing sites on a basis of combinatorial interactions among regulatory factors and pre-mRNA processing machinery [11]. The key to the formulation of the 'exon definition model' (Fig. 2a) was the finding that a downstream $5^{\prime}$ splice-site can stimulate a

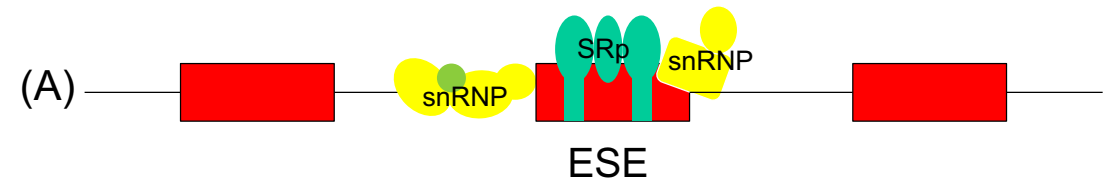

(B)

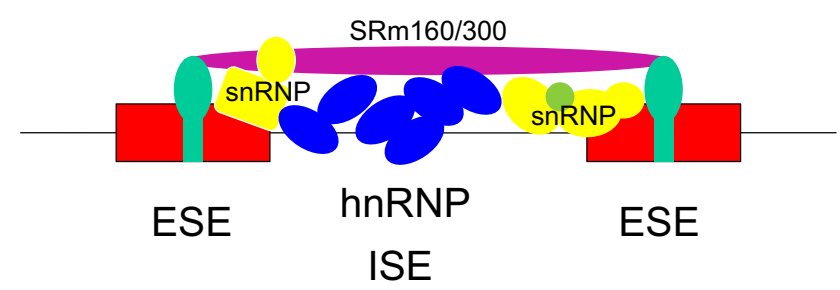

Fig. 2 Splice site localization in pre-mRNA via exon definition (a) or intron definition (b) models. a Splice site recognition in the exon definition model is enhanced across the exon by exon-bound proteins, e.g., SR proteins (SRp, green) bound to splicing enhancers (ESE), that interact with spliceosomal components (yellow) to stabilize their interaction with the pre-mRNA. Exons are shown as red boxes, introns as solid lines, and

ESEs as green boxes. b In the intron definition model, cross-intron interactions, e.g., mediated by hnRNP proteins (blue) bound to intronic splicing enhancers (ISE), or mediated by SRm160 or SRm300 proteins (purple), that interact with U1 snRNP, U2snRNP (yellow), and SR proteins (green), stabilize spliceosomal components (adapted from Soller [11]) 
weak upstream $3^{\prime}$ splice-site. Support for this model also comes from splicing stimulatory sequences termed exonic splicing enhancers (ESEs), which are preferentially bound by serine-arginine-rich (SR) proteins containing an RNA recognition motif (RRM) in addition to an arginine-serine-rich (RS) domain. Experimental and bioinformatics approaches have revealed a number of consensus sequences for ESEs that were also shown to be bound by SR proteins and are most prominently found in constitutive exons. Regulatory elements that antagonize exon definition are termed intronic or exonic splicing silencers (ISSs or ESSs) and are bound by negative regulators of splicing such as heterogeneous nuclear ribonucleoproteins (hnRNP). Since 5' and 3' splice sites come into proximity during spliceosome assembly through a number of RNA-protein and protein-protein interactions, the opposite 'intron definition model' has also been proposed (Fig. 2b). This model most likely applies for short introns. Spliceosomes are large and complex molecular machines found primarily within the nucleus. The spliceosome is assembled from small nuclear RNPs (snRNPs) and protein complexes. The spliceosome removes introns from a transcribed pre-mRNA, a kind of primary transcript.

What are serine/arginine-rich splicing factor family RNA-binding proteins and how do the factors function? Serine/arginine-rich splicing factors play an important role in constitutive and alternative splicing as well as during several steps of RNA metabolism. An important example is serine/arginine-rich splicing factor 1 (SFRS1) also known as alternative splicing factor 1 (ASF1), premRNA-splicing factor SF2 (SF2) or ASF1/SF2. It is an essential sequence-specific splicing factor involved in premRNA splicing [12]. SFRS1 is necessary for all splicing reactions to occur and influences splice-site selection in a concentration-dependent fashion. SFRS1 is also involved in mediating post-splicing activities, such as mRNA nuclear export and translation. The splicing regulator is in turn regulated by phosphorylation at the serines in its RS domain by the SR-specific protein kinase, SRPK1 [13]. Maslon et al. [14] have investigated the gene network that is translationally regulated by SRSF1. They performed a high-throughput deep sequencing analysis of polysomal fractions in cells overexpressing SRSF1. The work identified approximately 1,500 mRNAs that are translational targets of SRSF1. The mRNAs included mRNAs encoding proteins involved in cell cycle regulation, such as spindle, kinetochore, and $\mathrm{M}$ phase proteins, which are essential for accurate chromosome segregation. Their data indicate the rich landscape influenced by this splicing factor.

The contribution by Boddu et al. [10] underscores the fact why clinicians need to be aware of alternate splicing. More than $60 \%$ of all human disease-causing mutations may affect splicing rather than directly affecting coding sequences. One- third of all hereditary diseases are likely to have a splicing component [15]. Genome-wide analysis of alternative splicing is now performed routinely. Cross-linking and immunoprecipitation (CLIP) uses ultraviolet radiation to link proteins to RNA molecules in a tissue during splicing. A trans-acting splicing regulatory protein of interest is then precipitated using specific antibodies. When the RNA attached to that protein is isolated and cloned, the result reveals the target sequences for that protein. Another method for identifying RNA-binding proteins and mapping their binding to pre-mRNA transcripts is microarray evaluation of genomic aptamers by shift (MEGAshift). These technologies are upon us and we must know what they portend clinically.

Respectfully,

Friedrich C. Luft

\section{References}

1. Büscher R, Büscher AK, Weber S, Mohr J, Hegen B, Vester U, Hoyer PF (2013) Clinical manifestations of autosomal recessive polycystic kidney disease (ARPKD): kidney-related and non-kidney-related phenotypes. Pediatr Nephrol

2. Rossetti S, Harris PC (2007) Genotype-phenotype correlations in autosomal dominant and autosomal recessive polycystic kidney disease. J Am Soc Nephrol 18:1374-1380

3. Arbeiter A, Buscher R, Bonzel KE, Wingen AM, Vester U, Wohlschläger J, Zerres K, Nurnberger J, Bergmann C, Hoyer PF (2008) Nephrectomy in an autosomal recessive polycystic kidney disease (ARPKD) patient with rapid kidney enlargement and increased expression of EGFR. Nephrol Dial Transplant 23: 3026-3029

4. Bergmann C, Senderek J, Sedlacek B, Pegiazoglou I, Puglia P, Eggermann T, Rudnik-Schöneborn S, Furu L, Onuchic LF, De Baca $M$ et al (2003) Spectrum of mutations in the gene for autosomal recessive polycystic kidney disease (ARPKD/PKHD1). J Am Soc Nephrol 14:76-89

5. Bergmann C, Senderek J, Windelen E, Küpper F, Middeldorf I, Schneider F, Dornia C, Rudnik-Schöneborn S, Konrad M, Schmitt $\mathrm{CP}$ et al (2005) Clinical consequences of PKHD1 mutations in 164 patients with autosomale-recessive polycystic kidney disease (ARPKD). Kidney Int 67:829-848

6. Furu L, Onuchic LF, Gharavi A, Hou X, Esquivel EL, Nagasawa Y, Bergmann C, Senerek J, Zerres K, Germino GG et al (2003) Milder presentation of recessive polycystic kidney disease requires presence of amino acid substitution mutations. J Am Soc Nephrol 14:2004 2014

7. Black DL (2003) Mechanisms of alternative pre-messenger RNA splicing. Annu Rev Biochem 72:291-336

8. Wang ET, Sandberg R, Luo S, Khrebtukova I, Zhang L, Mayr C, Kingsmore SF, Schroth GP, Burge CB (2008) Alternative isoform regulation in human tissue transcriptomes. Nature 456(7221):470 476

9. Cognata VL, Iemmolo R, D’Agata V, Scuderi S, Drago F, Zappia M, Cavallaro S (2014) Increasing the coding potential of genomes through alternative splicing: the case of PARK2 gene. Curr Genomics 15(3):203-16

10. Boddu R, Yang C, O'Connor AK, Hendrickson RC, Boone B, Cui X, Garcia-Gonzalez M, Igarashi P, Onuchic LF, Germino GG, Guay- 
Woodford LM (2014) Intragenic motifs regulate the transcriptional complexity of Pkhd1/PKHD1. J Mol Med. doi:10.1007/s00109-0141185-7

11. Soller M (2006) Pre-messenger RNA processing and its regulation: a genomic perspective. Cell Mol Life Sci 63:796-819

12. Zuo P, Manley JL (1993) Functional domains of the human splicing factor ASF/SF2. EMBO J 12:4727-37

13. Hagopian JC, Ma CT, Meade BR, Albuquerque CP, Ngo JC, Ghosh G, Jennings PA, Fu XD, Adams JA (2008) Adaptable molecular interactions guide phosphorylation of the SR protein ASF/SF2 by SRPK1. J Mol Biol 382(4):894-909

14. Maslon MM, Heras SR, Bellora N, Eyras E, Cáceres JF (2014) The translational landscape of the splicing factor SRSF1 and its role in mitosis. ELife 3:e02028

15. Lim KH, Ferraris L, Filloux ME, Raphael BJ, Fairbrother WG (2011) Using positional distribution to identify splicing elements and predict pre-mRNA processing defects in human genes. Proc Natl Acad Sci U S A 108:11093-8 